\title{
On the relationship between environmental concerns and Low-Carbon behaviors of university students: Based on the investigation of $B$ University
}

\author{
S.Y. SHEN ${ }^{1}$ X.W. LIU ${ }^{2}$ \& Y.H. MA ${ }^{2}$ \\ ${ }^{1}$ School of Foreign Languages, Beihang University, Beijing, China \\ ${ }^{2}$ School of Public Administration, Beihang University, Beijing, China
}

KEYWORD: Undergraduates; Environmental Concerns; Pro-environmental Behaviors; Relationship ABSTRACT: The purpose of this paper is to analyze and measure the undergraduates' proenvironmental behaviors and environmental attitudes and the relationships between them as well. The study shows three kinds of attitude in terms of environmental concerns and the sequence decreases; regarding pro-environmental behaviors, the students behave better in private-area than the publicarea. Significant differences have been found in different gender, subjects, residence. The study has found the biospheric environmental concerns impact on the students' behaviors positively and significantly, and egoistic environment concerns impact on the students negatively significantly in both of the public and private area, moreover, altruistic environment concerns only have a positive impacts on private area behaviors.

\section{INTRODUCTION}

The Low-Carbon Economy under the perspective of Generalized Virtual Economy could satisfy people's material, social and psychological needs, it is a people oriented economy development mode. We have discussed its connotation and take eco-friendly city as example to explain it. However, we have less knowledge about individual Low-Carbon behavior (LCB) and its internal mechanism. We need to know the factors which would promote people's LCB and realize environmental and material values.

Since the 1960s, a series of environmental accidents and disasters triggered the constant development of environmental movement in western society. Meanwhile, they also promoted the masses' concern about the environmental problems and the conduct of LCB. The research of LCB and its influence factors have become a hot topic of social scientists. LCB is a term which is widely used by western researchers. In a cross-culture study, Hunter and his colleagues (2004) divided the LCB into public area LCB (such as joining in the environmental protection demonstration, donation) and private area LCB (such as purchasing green products, decreasing the utilization frequency of vehicle). Some Chinese scholars also followed this classification to examine the gender difference of LCB in China (Gong, 2008; Liu \& Wu, 2013).

Dunlap \& Jones (2002) defined environmental concern as the degree of people's awareness of environmental problems and support of resolving these problems or people's efforts to resolve these problems. Schultz \& Zelezny (1999) developed the Environmental Concern Scale (ECS) to measure people's abstract and general environmental concern. Researches have demonstrated the general applicability of ECS across culture and time. With regard to the relationship between environmental concern and environmental behaviors, however, there was not an accordant conclusion among researchers. Some researches indicated that environmental concern was a valid predictive factor of environmental behaviors, for example, the customers with higher level of environmental concern were more likely to purchase green products and had more LCB (Liu \& Wu, 2013).

China's Agenda 21 pointed out that young people were the significant impetus of environmental protection and sustainable development. University students were the nucleus of China's modernization construction and the main force of ecological civilization construction and they had the advantages of leading the harmonious coexistence of human and nature. Based on the important roles the university students played in current ecological civilization construction and low-carbon economic development in China, it was necessary to investigate and analyze the LCB and environmental concerns of university students. 


\section{METHOD}

\section{Participants}

1200 questionnaires were randomly dispensed at B universities in Beijing. 1158 questionnaires were returned and there were 1021 valid questionnaires (valid return rate was $88.2 \%$ ). $27.3 \%$ of the participants were female, $90.9 \%$ of them were Han. Urban, town and rural students accounted for $51.1 \%, 27.7 \%$ and $20.3 \%$ respectively. And $6.5 \%$ of participants' household income was below 1000 Yuan, and the percentage of participants whose household income ranged from 1001 to 4000 Yuan, 4001 to 7000 Yuan, 7001 to 10000 Yuan and above 10001 Yuan were $24.5 \%, 28.5 \%, 16.8 \%$ and $22.6 \%$ respectively. $70.8 \%$ of the students major in science and engineering, $29.2 \%$ of them major in liberal arts.

\section{Measures}

This research adopted the Chinese version of Environmental Concern Scale (ECS) revised by Liu \& $\mathrm{Wu}$ (2013) to measure participants' environmental concern. The revised ECS contained 12 items which were rated on a 5-point Likert scale. In Liu \& Wu's study, The internal consistency of the whole scale was 0.868 , and the internal consistency of three sub-scales ranged from 0.746 to 0.804 . In this study, coefficient of internal consistency of the whole scale was 0.910 , and coefficients of internal consistency of egoistic, altruistic and biospheric environmental concern were $0.874,0.833$ and 0.882 respectively.

The Public-Private LCB scale developed by Liu and Wu (2013) was adopted in this research. The scale consisted by 2 dimensions and contained 12 items which were rated on a 5-point Likert scale. In the previous study, the internal consistency of the whole scale was 0.768 . In this study, the coefficient of internal consistency of the whole scale was 0.798 , and coefficient of internal consistency of public and private area LCB were 0.738 and 0.714 respectively.

Besides the measures of environmental concern and LCB, we also surveyed students' social demography data, such as their university, college, discipline, gender, nationality, residence, average monthly income. Software of SPSS17.0 and LISREL8.17 were employed to analyze the questionnaire.

\section{RESULTS}

\section{The relationship between research variables and demographic factors}

The research examined the demographic differences of environmental concern. The results showed that there was significant gender difference on the score of biospheric environmental concern scale, the female students scored higher than male students. However, there was no significant gender, nationality, residence and income difference. The detailed results were shown in Table 1.

Table 1. Demographic difference of environmental concern.

\begin{tabular}{ccccc}
\hline \multirow{2}{*}{ gender } & & egoistic & altruistic & biospheric \\
& $t$ & -1.080 & -0.912 & -1.97 \\
discipline & $p$ & 0.281 & 0.362 & 0.049 \\
& $t$ & -0.214 & -0.283 & 1.911 \\
nationality & $F$ & 0.830 & 0.777 & 0.056 \\
& $F$ & -0.148 & 1.806 & 0.891 \\
residence & $F$ & 0.882 & 0.071 & 0.373 \\
\multirow{2}{*}{ income } & $F$ & 2.027 & 0.076 & 0.839 \\
& $F$ & 0.132 & 0.927 & 0.432 \\
& $p$ & 0.995 & 1.555 & 1.721 \\
\hline
\end{tabular}

The research also examined the demographic differences of LCB. The results showed that there were significant gender, discipline and residence difference on the score of two types of LCB. How- 
ever, there was no significant nationality and income difference. The detailed results were shown in Table 2.

Table 2. Demographic difference of LCB.

\begin{tabular}{cccc}
\hline & & public area & private area \\
\hline gender & $t$ & -4.650 & -4.669 \\
& $p$ & 0.000 & 0.000 \\
discipline & $t$ & -2.613 & -2.162 \\
& $p$ & 0.009 & 0.031 \\
nationality & $F$ & 0.774 & 0.294 \\
& $p$ & 0.439 & 0.769 \\
residence & $F$ & 3.573 & 4.478 \\
& $p$ & 0.028 & 0.012 \\
income & $F$ & 1.224 & 1.198 \\
& $p$ & 0.299 & 0.310 \\
\hline
\end{tabular}

To the gender variable, male students scored lower than female students on public and private area LCB. To the discipline variable, students major in liberal arts scored higher than those major in science and engineering on public and private area LCB. To different residence, the results of Turkey HSD indicated that rural students scored lower than urban and town students.

\section{Correlation Analysis}

Correlation analysis of research variables were shown in Table 3.

Table 3. Relative matrix of research variables

\begin{tabular}{lllll}
\hline & egoistic & altruistic & biospheric & \\
& & & \\
\hline altruistic & $0.651^{* *}$ & & & \\
biospheric & $0.445^{* *}$ & $0.602^{* *}$ & & \\
& & & & \\
public area & $0.065^{*}$ & $0.133^{* *}$ & $0.187^{* *}$ & $0.475^{* *}$ \\
private area & $0.138^{*}$ & $0.247^{* *}$ & $0.260^{* *}$ & 0.02 \\
& & & & \\
\hline $05, * * p<0.01$. & & & &
\end{tabular}

$* p<0.05, * * p<0.01$.

The results of correlation analysis indicated that three types of environmental concerns are significant correlated with two types of LCB. Such relationship showed that we could use Structure Equation Modeling (SEM) to explore the strength and path of the relationship between these variables. $\chi 2 / \mathrm{df}=13.86$, although such index higher than 5, considering the sample size are over 1000 , so this index would be accepted. To other indexes, NNFI $=0.90, \mathrm{CFI}=0.91, \mathrm{NFI}=0.90, \mathrm{RMSEA}=0.11$, all of them were accepted. Above all, the mode fitted the data well.

\section{SEM analysis of environmental concern and LCB}

Due to the limitation of traditional regression analysis in the research of multi-variables, we employed the SEM method to examine the relationship between the research variables. The path coefficients of 5 latent variables were shown in Table 4. It showed that egoistic and biospheric environmental concerns had direct effects on two types of LCB. Altruistic had a direct effect on private area LCB. 
Table 4. standardized path coefficient

\begin{tabular}{ccc}
\hline path & $\begin{array}{c}\text { standardized path } \\
\text { coefficient }\end{array}$ & $t$ \\
\hline biospheric to public & 0.42 & $8.98^{* * *}$ \\
biospheric to private & 0.49 & $8.32^{* * *}$ \\
egoistic to public & -0.16 & $-3.73^{* * *}$ \\
egoistic to private & -0.16 & $-2.54^{*}$ \\
altruistic to private & 0.20 & $2.28^{*}$ \\
\hline$*<0.05, * * p<0.01, * * * \mathrm{p}<0.001$. &
\end{tabular}

\section{Conclusion and implication}

\section{Conclusion}

This paper studied individual Low-Carbon behavior (LCB) and its internal mechanism, we draw the following conclusions.

First, altruistic environmental concern was the major environmental attitude among university students, and their biospheric concern level was low. Study results indicated that university students scored relatively higher on the whole ECS and detailed terms. However, the score on altruistic concern was higher than that on biospheric concern. This could be explained by Individual and Social orientation proposed by Yang (1993). People with individual orientation paid more attention to autonomy, emphasized the utilization and transformation of nature and social environment to satisfy their desires, interests and emotion. On the contrary, people with social orientation stressed the importance of involvement, which need people submit to and match up nature and social environment to construct and keep the harmonious relationship with the environment. Yang believed that Chinese people were typical social orientation.

Second, university students tend to express more private LCB. The results showed that university students LCB frequencies were low, and mainly express in private areas. However, they rarely participate in the organizational, public, interactive and wide-reaching public area LCB. On the one hand, although university students had higher level environmental concern, there would be many subjective and objective barriers when the environmental concern was put into specific LCB. On the other hand, many Chinese university students lived in the campus, hence, they seldom join in the public LCB and private LCB may be the major activities, such as the activities happened in their university dormitories. Furthermore, the public LCB relied on the organization's propaganda and guidance, so there were many work need to conduct to promote university students' public area LCB.

Third, students' score on environmental concern and LCB existed significant difference on several social demographic factors. To the gender variable, female students scored higher than male students on biospheric environmental concern, public and private LCB. This result was consistent with the study of Gong (2008), Liu \&Wu (2013). Hunter (2004) took the traditional gender role as the reason of this result. Individual behaviors and psychology were molded by the gender expect of cultural norms, in such framework, the traditional gender role of female was tender and caregiver who should be cooperative and sympathetic and should form the worldview of keeping various kinds of relationships. However, the traditional social role of male required them to provide economic supports to the family. Such role made males were more independent and dominant in pursuing the economic success. To the gender difference on private LCB, the reason might be limited free time dominance which determined that female's altruistic values tended to be expressed in the private areas (Gong, 2008). It was worthy to note that there was no significant difference exited between Han and minority's scores. This result was different from previous studies. The reason may be that the rapid development of the minority areas brought severe pollution to these areas, which made the raise of envi- 
ronmental concern. We should admit that the proportion of Han and minority in our study may influence the results.

Forth, the relation model of environmental concern and LCB could be accepted. This study employed related software to analyze the relationship between environmental concern and LCB. The fitness of the model reflected the causal relationship between different environmental concerns and specific LCB. The results showed that biospheric concern would positively influence two types of LCB, egoistic concern negatively influenced two types of LCB. However, altruistic concern only had a direct effect on the private area LCB.

\section{Implications}

Based on the above conclusions, we proposed that universities should take follow measures to promote university students' environmental concern and behaviors benefited to ecological civilization construction.

First, universities should guide students actively concern nature and the global ecological environment. In this study, we found that university students were concerned with environment on the whole. They pay attention to and avoid the harm of ecological environment destruction to people around them. Although it was a comforting result, it also reflected that students should enhance their attention to the harmonious relationship between human and nature environment and develop their global view. Therefore, universities and related government departments should not only advocate the idea of 'one for all, all for one', but also emphasize the idea of harmonious co-existence between human and nature and awaken people's respects to the nature and worship to the life in the progress of ecological civilization construction.

Second, the leading role of university students in ecological civilization construction should be encouraged. We found that university students have made positive efforts to the ecological civilization construction which reflected the good style and features of Chinese university students. However, compared with the score on environmental concern, students' score on LCB are lower which meant that the transformation from attitude to specific behaviors. Thus, universities should be persistent enough to advocate and encourage LCB and promote students to conduct conscious and active LCB. Moreover, we also found that the students' participation frequency of Public area LCB is low, universities should encourage students' leading role in ecological civilization construction and the discipline advantage and undertake the responsibility of advocating and setting example of LCB to the public.

Third, universities should persistently conduct diversified and hierarchical popularization activities of ecological civilization. In the study, we noticed that students of different gender, disciplines, residence scored differently on the research variables which required that universities should pay much more attention to the needs and affordability of ecological civilization construction of different groups of people and develop diversified ecological civilization construction activities. The relationship between environmental concern and LCB showed that different types of environmental concerns would have significant effects on students' LCB. Egoistic concern had a negatively significant effect on LCB which indicated that it would be difficult to promote university students' participation in ecological civilization construction if we just based on the personal perspective. Altruistic and biospheric concerns could positively influence LCB which would be the direction of universities' work. Universities should encourage students' altruistic and biospheric concerns and cultivate and guide students' hierarchical LCB through regular and organized group activities.

\section{Acknowledgements}

This work was financially supported by the Special Funds of the Generalized Virtual Economy Research [GX2010-1014 (Y)].

\section{References}

[1] Dunlap, R. E., \& R. E. Jones. 2002. Environmental Concern: Conceptual and Measurement Issues. In R. E. Dunlap and W. Michelson (eds), Handbook of Environmental Sociology: 482524. Westport, CT: Greenwood Press.

[2] Gong, W. J. 2008. A Gender Comparison on the Environment-friendly Behaviors of Contemporary Urban Residents. Journal of China University of Geosciences (Social Sciences Edition) 8(6): $37-42$. 
[3] Hunter, L. M., Hatch, A., \& Johnson, A. 2004. Cross-national gender variation in environmental behaviors. Social Science Quarterly 85(3): 677-694.

[4] Liu, X. W. \& Wu, J. P. 2013. Environmental Values and Pro-environmental Behaviors of College Students: Mediating Role of Environmental Concern. Studies of Psychology and Behavior 11(6): 780-785.

[5] Schultz, P. W., \& Zelezny, L. C. 1999. Values as predictors of environmental attitudes: Evidence for consistency across 14 countries. Journal of Environmental Psychology 19: 255-265. 